markedly elevated. A lateral cervical spine X-ray excluded bony pathology, but revealed pre-cervical soft tissue swelling. MRI showed extensive signal change in the odontoid peg, along with enhancement and fluid distension of the joint capsule - appearances in keeping with a diagnosis of septic arthritis of the atlanto-axial joint and osteomyelitis of the odontoid peg. The patient was commenced on once-daily intravenous antibiotics for six weeks, before switching to oral therapy for a total of three months. He responded well to treatment and recovered a full range of neck movements. Repeat imaging demonstrated resolution of inflammatory changes, and he was discharged from follow-up.

A high index of suspicion is required for the diagnosis of osteomyelitis, as it has variable presentation and initial investigations may be misleading. This case indicates that nuclear imaging should be done as a matter of urgency in a child who has no appreciable active or passive neck movement to rule out bony pathology, and that although a rare cause of stiff neck in children, odontoid peg osteomyelitis should be considered as a differential diagnosis even in a child who does not look septic. This case also illustrates that odontoid peg osteomyelitis may subsequently lead to septic arthritis of the atlantoaxial joint, which has not been previously described in the literature.

\section{G453(P) MANAGEMENT OF KAWASAKI DISEASE IN A DISTRICT GENERAL HOSPITAL. IS THERE AN OPTIUM ASPIRIN DOSE?}

K Murtagh, F Damda. Department of Paediatrics, University Hospital Lewisham, Lewisham and Greenwich NHS Trust, London, UK

10.1136/archdischild-2015-308599.407

Aims Kawasaki disease (KD) is the commonest cause of paediatric acquired heart disease in the UK. Early treatment significantly reduces coronary artery complications. This study aimed to identify cases of $\mathrm{KD}$ presenting to our district general hospital (DGH). We wished to see how well diagnosis and management adhered to local guidelines.

Methods Retrospective review of case notes of patients admitted to our district general and diagnosed with KD between October 2011 and October 2014. Diagnosis and management was compared to local guideline.

Results Eighteen KD patients of variable age (3 months to 9 years) and ethnicity were identified. Two thirds of cases had incomplete KD. All had a history of fever $>5$ days. Diagnostic features included conjunctivitis (16/18); mucosal changes (15/ $18)$; rash (11/18); cervical lymphadenopathy and peripheral extremity changes (8/18). Irritability was a common feature. BCG scar changes were also present (2/18).

All cases received intravenous immunoglobulin (IVIG) at $2 \mathrm{~g} /$ $\mathrm{kg}$ and high dose aspirin at $30-100 \mathrm{mg} / \mathrm{kg} /$ day except one who received aspirin alone as afebrile at presentation.

Five patients had suspicion of coronary artery dilatation/ prominence on initial echocardiogram. Three subsequently developed coronary artery dilatation all of whom had received IVIG during first week of fever.

Three cases were transferred to tertiary centre for secondline management encompassing repeat IVIG (3/18), infliximab $(2 / 18)$ and corticosteroids (1/18).
Conclusion Two thirds of KD cases were incomplete. Ninetyfour percent had IVIG. Inflammatory markers, laboratory criteria and BCG scar change did not correspond with cardiovascular complications. Dose of aspirin given varied between $30-100 \mathrm{mg} / \mathrm{kg} /$ day depending on tertiary centre recommendation. All patients were managed appropriately. Those that failed to respond were referred to a tertiary centre. This study demonstrates most KD can be managed locally with adherence to clear guidelines and receiving tertiary advice when required. However clearer consensus on high-dose aspirin is needed.

\section{G454(P) RETROSPECTIVE SURVEY OF CHILD PROTECTION CASE CHARACTERISTICS IN AN ACUTE AND COMMUNITY SETTING}

R Sharma, P Mistry. Community Paediatric Department, University Hospitals of Leicester, Leicester, UK

\subsection{6/archdischild-2015-308599.408}

Aim The first principle of safeguarding services stated in the Munro report $2010^{1}$ places the quality of experience and views of the child at the centre. Lord Laming's review in $2009^{2}$ recommended minimum requirements to be met during the safeguarding process. Safeguarding assessments for children occur in both an outpatient (OP) clinic setting and in an acute out of hours $(\mathrm{OOH})$ setting. The aim of this survey was to compare the number, type and outcome of safeguarding assessments between these two settings.

Method Retrospective case-notes review of all cases where a safeguarding examination was completed due to Section 47 concerns from January to March 2013. Those that were referred due to accidental incidents or where there was found to be no safeguarding concern were excluded. Data was obtained on documentation of the process and timeline for assessment as well as the types of case assessed. Outcomes one year later included children remaining with biological family and children remaining on a child protection plan.

Results A total of 67 safeguarding cases were seen during that period of time. $78 \%$ were seen in an OP setting. Median age $=5$ yrs 7 months (IQR 2 to 9 years). $\mathrm{OOH}$ median age $=20$ months (IQR -12 to 35 months). Median waiting times and type of abuse were similar. Twenty eight percent were on a child protection plan (CPP) at the time of referral to the OP service versus $9 \%$ for $\mathrm{OOH}$ referrals. Fractures and burns were more often seen in the $\mathrm{OOH}$ setting whereas no injury seen was more often documented in the OP setting.

Conclusions The type of safeguarding cases seen do differ between acute and community settings in the age of presentation, the type of injury seen, the presence of history taking from the child and the numbers on a child protection plan at the point of referral. However the type of abuse, whether physical, neglect or sexual is similar. Further work needs to be done on standardising safeguarding practice across acute and community settings.

\section{REFERENCES}

1 The Munro Review of Child protection. Department of Education. 2011

2 The Protection of Children in England: A Progress Report. Department of Education. 2009 
G455(P) IMPROVING ACCESS AND QUALITY OF DIAGNOSTIC SERVICES IN A DEVELOPMENTAL AND BEHAVIOURAL SERVICE FOR PRE-SCHOOL CHILDREN - THE TRIAGETRACK MODEL

S Singhal, HH Lim, AK Chua, LM Daniel, SB Lim. Department of Child Development, KK Women's and Children's Hospital, Singapore

10.1136/archdischild-2015-308599.409

Aim The Department of Child Development (DCD) at our hospital is the major diagnostic and interim intervention service provider for pre-school children with developmental and behavioural disorders in our country. With increased public awareness and emphasis on early detection, the demand for our services has risen tremendously. This was not met with the traditional medical model of multi-disciplinary service delivery. We implemented a novel triage-track inter-disciplinary service model to enhance access and quality of services. This paper presents our 4-year experience with the triage-track model from June 2010.

Methodology Through cause and effect analysis, a triage workgroup identified progressive solutions to improve case-differentiation and service prioritisation. The measures streamlined and defined were: 1) secondary screening and case management pathways in the triage clinic, 2) tertiary diagnostic and interim intervention model for specialised tracks [Learning and Behaviour (LB) track, Autism Spectrum Disorder (ASD) track, and Complex track], 3) documentation standards for continuity of care, 4) interdisciplinary professional roles which promoted cross-disciplinary learning. We conducted three Plan-Do-StudyAction (PDSA) cycles (Oct 2010-Nov 2012) before embarking on installation (Dec 2012- Mar 2013) and full implementation (since April 2013).

Results The average monthly wait-time (interval between primary care referral to first DCD visit) improved by $84.2 \%$ with the triage-track model [pre-implementation 139 days (June 2010) versus full-implementation 22 days (June 2014)]. In 2012, the triage-track model improved operational capacity by $67.9 \%$ compared to the Traditional model [4.7 patients/triageclinic session $(n=1355)$ versus 2.8 patients/traditional new-case session ( $n=799)]$. In 2013, 32.2\% patients were referred to the specialised tracks for tertiary diagnostic evaluation and management (ASD: 19.2\%; LB: 11.7\%; Complex: 1.3\%). The ASD track achieved significant improvement in wait-time and cycletime for ASD diagnostic evaluation and family-centeredness of services. The LB track enhanced comprehensiveness of diagnostic evaluation and fast-tracked $21 \%$ of evaluations for patients. In the complex track, all patients completed full evaluation as targeted within a 6-month period.

Conclusion The interdisciplinary triage-track model improved access to services, enhanced operational capacity and quality of care in our centre.

\section{G456(P) IMPROVING THE DIAGNOSTIC PROCESS AND DELIVERY OF INTERIM INTERVENTION FOR PRE-SCHOOL AUTISM SPECTRUM DISORDER CHILDREN IN A DEVELOPMENTAL CLINIC}

SB Lim, HH Lim, CM Wong, S Singhal, PH Yang, KC Wong, S Amir. Department of Child Development, KK Women's and Children's Hospital, Singapore

\subsection{6/archdischild-2015-308599.410}

Introduction Autism Spectrum Disorder (ASD) is increasingly common, and the department we work in is a key diagnostic and interim service provider for ASD among pre-school children. To improve the diagnostic process and delivery of interim intervention services for children and families referred to our department for ASD, we established an interdisciplinary ASD team, which initiated a Clinical Practice Improvement Project (CPIP) in September 2007 to provide an "ASD track" service. In this paper, we share our learning from this project.

Methods The ASD team analysed the root cause through cause and effect analysis. We derived solutions which improved 1) case differentiation mechanism and prioritisation of services, 2) standardisation of diagnostic processes and documentation, 3) continuity of care and case management, 4) cross-disciplinary training and professional standards, 5) family-centeredness of our interim intervention services. We conducted 3 Plan-DoCheck-Action (PDCA) cycles (January 2010-April 2011) before implementing the ASD track service in May 2011.

Results This paper presents results of our implementation. From September 2011 to March 2012, 181 cases were referred to the ASD team from the DCD triage clinic. Of these, 93 were classical ASD and offered the fast-track diagnostic service, for which $73(78.5 \%)$ underwent 2-hour psychological assessment.

\section{Improvements of ASD track from old process}

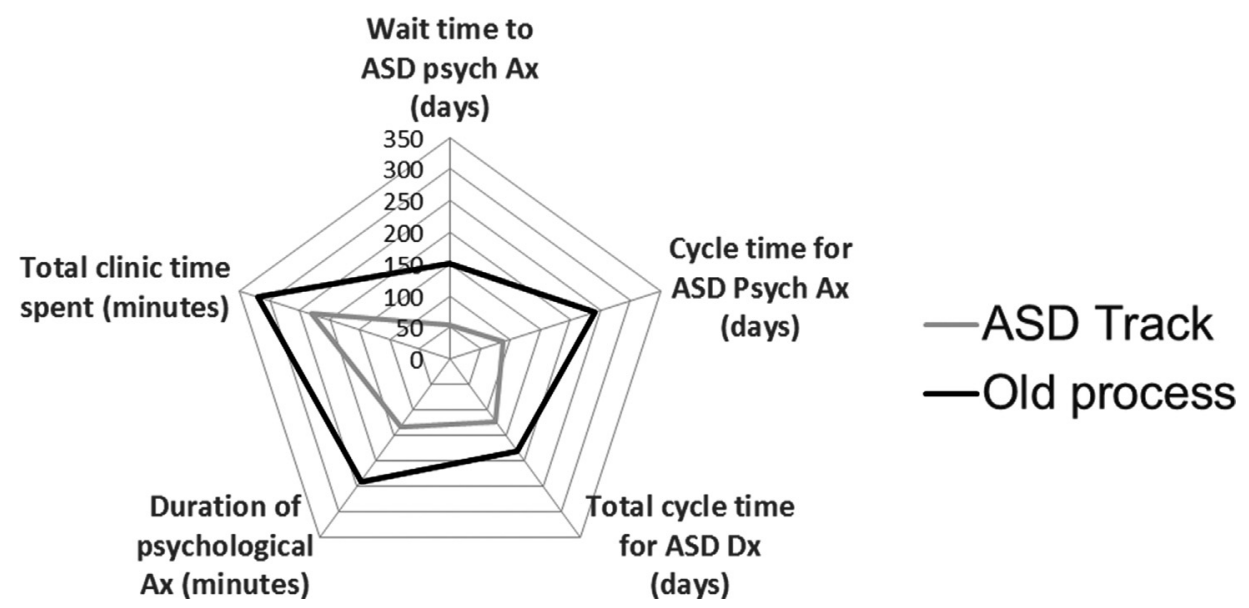

